\title{
Association of a Common G6PC2 Variant with Fasting Plasma Glucose Levels in Non-Diabetic Individuals
}

\author{
F.Y. Demirci ${ }^{a}$ A.S. Dressen ${ }^{a}$ R.F. Hamman ${ }^{c}$ C.H.Bunker ${ }^{\text {b }}$ C.M. Kammerer ${ }^{\text {a }}$ \\ M.I. Kamboha \\ Departments of a Human Genetics and ${ }^{b}$ Epidemiology, Graduate School of Public Health, University of Pittsburgh, \\ Pittsburgh, Pa., 'Department of Preventive Medicine and Biometrics, University of Colorado Denver, Aurora, Colo., \\ USA
}

\section{Key Words}

Blood glucose $\cdot$ Plasma glucose $\cdot$ Fasting plasma glucose $\cdot$ G6PC2 - Single nucleotide polymorphism - Polymorphism • rs560887

\begin{abstract}
Background/Aims: Fasting plasma glucose (FPG) levels correlate with cardiovascular disease and mortality in both diabetic and non-diabetic subjects. G6PC2 encodes a pancreatic islet-specific glucose-6-phosphatase-related protein and G6pc2-null mice were reported to exhibit decreased blood glucose levels. Two recent genome-wide association studies have implicated a role for G6PC2 in regulation of FPG levels in the general European population and reported the strongest association with the rs560887 SNP. The purpose of this study was to replicate this association in our independent epidemiological samples. Methods: DNA samples from non-Hispanic white Americans (NHWs; $n=623$ ), Hispanic Americans $(n=410)$ and black Africans $(n=787)$ were genotyped for rs560887 using TaqMan allelic discrimination. Results: While no minor allele A of rs560887 was observed among blacks, its frequency was $33 \%$ in NHWs and $17.5 \%$ in Hispanics. The rs560887 minor allele was associated with reduced FPG levels in non-diabetic NHWs $(p=0.002$ under an additive model). A similar trend of association was
\end{abstract}

observed in non-diabetic Hispanics ( $p=0.076$ under a dominant model), which was more pronounced in normoglycemic subjects $(p=0.036)$. Conclusions: Our results independently confirm the robust association of G6PC2/rs560887 with FPG levels in non-diabetic NHWs. The observed evidence for association in Hispanics warrants further studies in larger samples.

Copyright $\odot 2009$ S. Karger AG, Basel

\section{Introduction}

Blood glucose levels are maintained within narrow limits by hormonal and other regulators to prevent the harmful effects of hypo- or hyperglycemia. Acquired factors such as age and obesity appear to contribute to, but cannot fully explain, the inter-individual variation in blood glucose concentration [1]. Fasting plasma glucose (FPG) levels are under significant genetic influence (heritability estimates $>25 \%$ ) and have been shown to correlate with cardiovascular disease and mortality in both diabetic and non-diabetic individuals [2-8].

Glucose-6-phosphatase catalytic 2 gene (G6PC2, also known as IGRP, on chromosome $2 \mathrm{q} 24.3$ ) encodes a pancreatic islet-specific glucose-6-phosphatase cataly tic subunit-related protein. G6PC2 had initially drawn attention

\section{KARGER \\ Fax +41613061234 E-Mail karger@karger.ch} www.karger.com
() 2009 S. Karger AG, Basel 0250-6807/10/0561-0059\$26.00/0

Accessible online at:

www.karger.com/anm
F. Yesim Demirci, MD

Department of Human Genetics, GSPH

University of Pittsburgh

Pittsburgh, PA 15261 (USA)

Tel. +1 412624 3066, Fax +1 412383 7844, E-Mail fyd1@ pitt.edu 
Table 1. Biometric and quantitative data of 2 non-diabetic US samples; non-Hispanic whites (NHWs) and Hispanics

\begin{tabular}{|c|c|c|c|c|c|c|}
\hline & \multicolumn{3}{|l|}{ NHWs } & \multicolumn{3}{|l|}{ Hispanics } \\
\hline & $\begin{array}{l}\text { female } \\
(n=328)\end{array}$ & $\begin{array}{l}\text { male } \\
(\mathrm{n}=295)\end{array}$ & $\begin{array}{l}\text { all }^{\mathrm{a}} \\
(\mathrm{n}=623)\end{array}$ & $\begin{array}{l}\text { female } \\
(n=208)\end{array}$ & $\begin{array}{l}\text { male } \\
(n=202)\end{array}$ & $\begin{array}{l}\text { all }^{\mathrm{a}} \\
(\mathrm{n}=410)\end{array}$ \\
\hline Age, years & $52.67 \pm 0.64$ & $53.01 \pm 0.66$ & $52.83 \pm 0.46$ & $50.18 \pm 0.87$ & $52.12 \pm 0.88$ & $51.13 \pm 0.62$ \\
\hline $\mathrm{BMI}, \mathrm{kg} / \mathrm{m}^{2}$ & $24.81 \pm 0.24$ & $26.27 \pm 0.19$ & $25.50 \pm 0.16$ & $25.89 \pm 0.34$ & $25.55 \pm 0.28$ & $25.72 \pm 0.22$ \\
\hline Fasting glucose, $\mathrm{mg} / \mathrm{dl}^{\mathrm{b}}$ & $93.35 \pm 0.46$ & $98.16 \pm 0.47$ & $95.63 \pm 0.34$ & $93.26 \pm 0.58$ & $97.91 \pm 0.62$ & $95.55 \pm 0.44$ \\
\hline Normal (70-99 mg/dl) & $81.7 \%$ & $60 \%$ & $71.4 \%$ & $79.8 \%$ & $63.2 \%$ & $71.6 \%$ \\
\hline Impaired (100-125 mg/dl) & $18.3 \%$ & $40 \%$ & $28.6 \%$ & $20.2 \%$ & $36.8 \%$ & $28.4 \%$ \\
\hline Fasting insulin, $\mu \mathrm{U} / \mathrm{ml}$ & $10.86 \pm 0.38$ & $12.19 \pm 0.41$ & $11.49 \pm 0.28$ & $13.78 \pm 0.59$ & $12.95 \pm 0.53$ & $13.37 \pm 0.40$ \\
\hline Total cholesterol, mg/dl & $218.23 \pm 2.43$ & $213.74 \pm 2.27$ & $216.11 \pm 1.67$ & $211.54 \pm 2.88$ & $213.89 \pm 3.24$ & $212.70 \pm 2.16$ \\
\hline LDL-C, mg/dl & $135.52 \pm 2.17$ & $140.39 \pm 2.06$ & $137.80 \pm 1.51$ & $130.54 \pm 2.54$ & $138.18 \pm 3.05$ & $134.28 \pm 1.98$ \\
\hline $\mathrm{HDL}-\mathrm{C}, \mathrm{mg} / \mathrm{dl}$ & $56.84 \pm 0.80$ & $44.00 \pm 0.63$ & $50.76 \pm 0.57$ & $52.28 \pm 0.94$ & $45.23 \pm 0.83$ & $48.81 \pm 0.65$ \\
\hline Triglycerides, mg/dl & $130.75 \pm 3.41$ & $149.15 \pm 4.45$ & $139.45 \pm 2.79$ & $142.02 \pm 4.52$ & $156.26 \pm 5.23$ & $149.03 \pm 3.47$ \\
\hline
\end{tabular}

Data are unadjusted means \pm SE after removal of outliers.

${ }^{a}$ Including subjects with missing data or outliers for some of the variables.

${ }^{b}$ SI conversion factor for fasting glucose (from $\mathrm{mg} / \mathrm{dl}$ to $\mathrm{mmol} / \mathrm{l}$ ): divide by 18 or multiply by 0.0555 . SI conversion factors for other quantitative traits can be found at http://jama.ama-assn.org/content/vol295/issue1/images/data/103/DC6/JAMA_auinst_si.dtl.

as a major autoantigen for $\mathrm{T}$ cells that play an essential role in the development of type 1 diabetes in non-obese mice and humans [9-11]. G6pc2-null mice exhibit a mild metabolic phenotype with decreased blood glucose levels but unchanged insulin, glucagon, glycerol and cholesterol concentrations [12]. Recently, 2 genome-wide association studies (GWAS) have implicated a role for G6PC2 in contributing to inter-individual variation in FPG levels in the general population $[13,14]$. Both GWAS reported G6PC2/rs560887 [G>A, minor allele frequency (MAF) = 0.30 in Europeans] as the SNP most strongly associated with FPG levels among non-diabetic Europeans.

As replication of new findings by independent groups in independent samples is crucial for establishing the genetic associations for complex diseases, we sought to replicate the association of G6PC2/rs560887 with FPG levels in independent samples of non-Hispanic white (NHW) and Hispanic Americans. We also tried to assess the effect of rs560887 in black Africans, but the SNP turned out to be monomorphic in our sample.

\section{Subjects and Methods}

\section{Subjects}

The study samples consisted of 623 NHWs and 410 Hispanics who were non-diabetic unrelated Americans from the San Luis Valley Diabetes Study, a population-based case-control study of type 2 diabetes (T2D) in the San Luis Valley, Southern Colorado
[15-18]. A standard oral glucose tolerance test and World Health Organization criteria (www.who.int/topics/diabetes_mellitus/ en/) were used to identify the subjects with normal glucose tolerance, impaired glucose tolerance [2-hour oral glucose tolerance test plasma glucose levels of 140-199 mg/dl (7.8-11 mmol/l)], or previously unknown diabetes. Individuals with FPG levels below $100 \mathrm{mg} / \mathrm{dl}(5.6 \mathrm{mmol} / \mathrm{l})$ were considered normoglycemic, those with levels of $100-125 \mathrm{mg} / \mathrm{dl}(5.6-6.9 \mathrm{mmol} / \mathrm{l})$ were considered to have impaired fasting glucose, and those with levels of $126 \mathrm{mg} /$ $\mathrm{dl}(7 \mathrm{mmol} / \mathrm{l})$ and above were considered to have diabetes [19]. Non-diabetic individuals [blood glucose levels below $126 \mathrm{mg} / \mathrm{dl}$ (7 mmol/l)] included normoglycemic subjects as well as those with impaired glucose levels. The present study included a subset of non-diabetic participants; although some individuals had impaired fasting glucose, all subjects had normal glucose tolerance test results. The demographic features and characteristics of NHWs and Hispanics are presented in table 1. Detailed information on the black African sample (from Benin City, Nigeria), from which 787 individuals were included in this study, can be found elsewhere [20]. The study was approved by the University of Pittsburgh Institutional Review Board.

\section{Laboratory Methods}

Blood samples were drawn after overnight fasting. Glucose concentration was determined using glucose oxidase method and total immunoreactive insulin concentration using double-antibody radioimmunoassay [15-18]. Total cholesterol, HDL cholesterol, and triglycerides were measured using enzymatic methods, and LDL cholesterol was calculated by the Friedewald equation when triglyceride levels were less than $400 \mathrm{mg} / \mathrm{dl}(4.5 \mathrm{mmol} / \mathrm{l})$ [15-18]. 
Table 2. Adjusted ${ }^{\mathrm{a}}$ mean values $\pm \mathrm{SE}$ of quantitative metabolic traits among three G6PC2/rs560887 genotypes in 2 non-diabetic US populations; non-Hispanic whites (NHWs) and Hispanics

\begin{tabular}{|c|c|c|c|c|c|c|c|c|}
\hline & \multicolumn{4}{|l|}{ NHWs } & \multicolumn{4}{|l|}{ Hispanics } \\
\hline & \multicolumn{4}{|c|}{ G6PC2/rs560887 genotypes } & \multicolumn{4}{|c|}{ G6PC2/rs560887 genotypes } \\
\hline & GG & GA & AA & $\mathrm{p}$ value $\mathrm{e}^{\mathrm{e}}$ & GG & GA & AA & $p$ value $^{f}$ \\
\hline Genotype count ${ }^{\mathrm{b}}$ & 270 & 291 & 61 & & 278 & 109 & 16 & \\
\hline Fasting glucose, $\mathrm{mg} / \mathrm{dl}^{\mathrm{c}}$ & $96.50 \pm 0.49$ & $95.50 \pm 0.45$ & $92.94 \pm 1.00$ & 0.002 & $96.02 \pm 0.46$ & $94.34 \pm 0.87$ & $95.33 \pm 2.11$ & 0.076 \\
\hline Fasting insulin, $\mu \mathrm{U} / \mathrm{ml}^{\mathrm{d}}$ & $11.95 \pm 0.45$ & $11.23 \pm 0.34$ & $10.66 \pm 0.66$ & 0.199 & $13.15 \pm 0.39$ & $13.99 \pm 0.68$ & $11.98 \pm 0.76$ & 0.267 \\
\hline Total cholesterol, mg/dl & $218.59 \pm 2.43$ & $214.10 \pm 2.36$ & $216.74 \pm 4.95$ & 0.363 & $213.90 \pm 2.69$ & $206.94 \pm 3.53$ & $229.06 \pm 10.75$ & 0.378 \\
\hline LDL-C, mg/dl & $140.72 \pm 2.30$ & $134.88 \pm 2.08$ & $140.39 \pm 4.63$ & 0.317 & $134.72 \pm 2.49$ & $130.00 \pm 3.28$ & $152.07 \pm 10.11$ & 0.668 \\
\hline $\mathrm{HDL}-\mathrm{C}, \mathrm{mg} / \mathrm{dl}^{\mathrm{d}}$ & $51.00 \pm 0.74$ & $50.63 \pm 0.73$ & $50.15 \pm 1.48$ & 0.737 & $49.23 \pm 0.73$ & $47.92 \pm 1.09$ & $48.12 \pm 2.67$ & 0.379 \\
\hline Triglycerides, $\mathrm{mg} / \mathrm{dl}^{\mathrm{d}}$ & $135.49 \pm 3.73$ & $145.45 \pm 4.32$ & $130.51 \pm 7.19$ & 0.644 & $150.59 \pm 3.97$ & $146.35 \pm 6.98$ & $144.28 \pm 12.53$ & 0.339 \\
\hline $\mathrm{BMI}^{\mathrm{d}}, \mathrm{kg} / \mathrm{m}^{2}$ & $25.33 \pm 0.23$ & $25.50 \pm 0.23$ & $26.36 \pm 0.57$ & 0.145 & $25.59 \pm 0.25$ & $26.19 \pm 0.47$ & $25.37 \pm 0.77$ & 0.368 \\
\hline
\end{tabular}

${ }^{a}$ BMI adjusted for 'gender, age and smoking' and all other traits adjusted for 'gender, age, BMI and smoking'.

${ }^{b}$ Including subjects with missing data or outliers for some of the variables.

c SI conversion factor for fasting glucose (from $\mathrm{mg} / \mathrm{dl}$ to $\mathrm{mmol} / \mathrm{l})$ : divide by 18 or multiply by 0.0555 . SI conversion factors for other quantitative traits can be found at http://jama.ama-

assn.org/content/vol295/issue1/images/data/103/DC6/JAMA_ auinst_si.dtl.

${ }^{\mathrm{d}}$ Natural log-transformed before analysis.

${ }^{\mathrm{e}} \mathrm{p}$ value under the additive model, adjusted for the same covariates used for adjusting the mean values.

${ }^{\mathrm{f}} \mathrm{p}$ value under the dominant model, adjusted for the same covariates used for adjusting the mean values.

\section{Genotyping}

DNA samples were genotyped for rs560887 using a TaqMan SNP genotyping assay (C____323082_10; Applied Biosystems, Foster City, Calif., USA) and end-point fluorescence readings were performed on an ABI Prism 7900HT instrument (Applied Biosystems). The overall genotype call rate was $99.8 \%$ in NHWs, $98.3 \%$ in Hispanics, and $98.5 \%$ in blacks. The discrepancy rate was $0 \%$ in all samples for $10 \%$ random repeats.

\section{Statistical Methods}

Allele and genotype frequencies were determined by direct counting. Deviation of the observed genotype frequencies from those predicted by Hardy-Weinberg equilibrium was evaluated using the $\chi^{2}$ goodness-of-fit test. Dependent quantitative variables with skewed distributions were transformed (using log transformation) to reduce the effects of non-normality. After initial removal of extreme outliers, additional outliers falling outside of the transformed mean $\pm 3.5 \mathrm{SD}$ range were excluded in each population, separately in males and females. This excluded a maximum of 4 values for any trait in any population sample. Analysis of variance and linear regression were used to test for the effects of genotypes on the quantitative traits transformed (if necessary) and adjusted for 4 covariates (gender, age, BMI and smoking) included in the model. When BMI was treated as a dependent variable, 3 covariates (gender, age, and smoking) were used in the model. Genotype associations were tested under the 'additive' model in NHWs in order to replicate the findings of original GWAS in Europeans. Due to the smaller size of the Hispanic sample, the tests were performed under the 'dominant' model in this sample. All computations were performed using the R statistical software (version 2.3.1; www.r-project.org). Because we were test- ing a specific hypothesis regarding the relationship between the G6PC2 genotypes and FPG levels and other metabolic traits, a nominal $p$ value of less than $5 \%$ was considered as evidence of association.

\section{Results}

The rs560887 genotype frequencies were in HardyWeinberg equilibrium and the frequency of the minor allele $A$ was $33 \%$ in NHWs (similar to that previously reported for Europeans in the literature and dbSNP; www. ncbi.nlm.nih.gov/sites/entrez?db=snp) and less common (17.5\%) in Hispanics. Data from 775 successfully genotyped black Africans did not show any example of allele $A$, suggesting that if it exists in this population, its frequency is extremely low. It is unlikely that heterozygotes in black Africans were overlooked as a result of technical issues since the TaqMan genotype clusters were compact and well-separated. Among the 3 submissions in the dbSNP, 2 submitters reported a MAF of 0 or $0.8 \%$ in 'black Africans' while 1 reported a higher MAF of $4.3 \%$ in 'black Americans'; the latter is most likely due to Caucasian admixture.

Table 2 summarizes the association analysis results for FPG levels and other quantitative metabolic traits in 
'non-diabetic' NHWs and Hispanics. G6PC2/rs560887 minor allele was significantly associated with reduced FPG levels in NHWs after adjusting for gender, age, BMI and smoking $(\mathrm{p}=0.002$ under an additive model; major allele homozygote $=0$, heterozygote $=1$, minor allele homozygote $=2$ ). The effect size of rs560887 was small and explained only $\sim 1.4 \%$ of the variation in FPG levels. Both the direction and size of this effect were consistent with GWAS in Europeans. We observed a similar relationship between G6PC2/rs560887 and FPG levels in 'non-diabetic' Hispanics, however, it was not statistically significant (adjusted $\mathrm{p}=0.076$ under a dominant model; major allele homozygotes vs. heterozygotes + minor allele homozygotes). When the association with FPG levels was tested in only 'normoglycemic' subjects (by excluding non-diabetic individuals with impaired fasting glucose levels; table 1), it did not improve in NHWs (data not shown), however, it became modestly significant in Hispanics (adjusted $\mathrm{p}=0.036$ under the dominant model; adjusted mean values $\pm \mathrm{SE}$ were $91.54 \pm 0.37$ for GG vs. $90.18 \pm$ 0.51 for GA + AA). Using the same genetic models (additive in NHWs and dominant in Hispanics), we next tested whether G6PC2/rs560887 was associated with other quantitative traits in non-diabetic NHWs and Hispanics. We did not observe a significant association with BMI, fasting insulin levels or lipid measurements in either sample.

\section{Discussion}

Epidemiological studies suggested a relationship between blood glucose levels and risk for cardiovascular disease and all-cause mortality in both diabetic and nondiabetic subjects [2-8]. A systematic overview and metaanalysis of non-diabetic cohort studies revealed a graded relationship between glucose levels and cardiovascular risk, extending below the diabetic threshold [6]. A Ushaped relation between glucose levels and mortality was reported [7]; that is, both high (diabetes or impaired glucose levels) and low [ $<70$ or $80 \mathrm{mg} / \mathrm{dl}$ (3.9 or $4.4 \mathrm{mmol} / \mathrm{l})$ ] FPG levels were associated with increased mortality risk from cardiovascular disease and all causes. The DECODE study [8] also reported increased mortality risk with both high and very low glucose concentrations. These observations highlight the importance of investigating not only the genetic determinants of T2D but also those involved in regulation of FPG levels in non-diabetic individuals.
Two recent GWAS reported an association of small effect size $(\sim 1 \%)$ between the G6PC2/rs560887 SNP and reduced FPG levels in the general European population $[13,14]$. The first GWAS [13] evaluated 392,935 SNPs in a discovery sample of 654 'normoglycemic' French subjects and was able to replicate the strongest association observed with FPG levels (G6PC2/rs560887; p $=4 \times 10^{-7}$ adjusted for age, gender and BMI under the additive model) in additional French and Finnish samples. The second GWAS [14] investigated the SNPs from 2 genomewide scans in a total of 5,088 'non-diabetic' individuals from Finland and Sardinia (Italy) and found a strong association between FPG levels and a SNP (rs563694) that resides between G6PC2 and $A B C B 11\left(\mathrm{p}=3.5 \times 10^{-7}\right)$. The same study [14] was able to confirm the significant association of rs563694 in 6 of 7 follow-up samples of mixed European descent. Subsequent genotyping/analyses of the G6PC2-ABCB11 region by the second GWAS [14] also revealed that G6PC2/rs560887 [in high linkage disequilibrium (LD) with $\mathrm{rs563694} D^{\prime}=0.99, r^{2}=0.84$ ] showed the strongest association with fasting glucose $\left(\right.$ meta $\left.\mathrm{p}=2.8 \times 10^{-10}\right)$. Interestingly, rs560887 did not show evidence of association with T2D in either GWAS $[13,14]$, leading the authors to hypothesize that either the genetic determinants of FPG levels in physiological states are different than those associated with T2D risk or the effect of G6PC2 variation on T2D susceptibility is too small to be easily detected.

Our results in 'non-diabetic' NHWs support the findings of recent GWAS with a significant association (adjusted $\mathrm{p}=0.002$ under the additive model) of G6PC2/ rs560887 with FPG levels in the same direction (minor allele $A$ associated with reduced FPG levels). Consistent with GWAS, the effect size of rs560887 was small and accounted for only $\sim 1.4 \%$ of inter-individual variation in FPG levels. Although it was not statistically significant, we observed a similar trend of association in 'nondiabetic' Hispanics (adjusted $\mathrm{p}=0.076$ under the dominant model), which was more pronounced and modestly significant when analyzed only in 'normoglycemic' subjects after excluding non-diabetic individuals with impaired fasting glucose levels (adjusted $p=0.036$ under the dominant model). It is important to note that we had less power to detect the association in Hispanics due to the smaller sample size (410 Hispanics vs. $623 \mathrm{NHWs)}$ and lower MAF of rs560887 (17.5\% in Hispanics vs. 33\% in NHWs). In addition, there may be LD structure differences between the two ethnic groups; such that the true causative genetic variant(s) may be in high LD with rs560887 in NHWs but in low LD in Hispanics. Consis- 
tent with recent GWAS and mouse studies, we did not detect significant association with BMI, fasting insulin levels, or lipid measurements in either population, suggesting that the observed association with FPG levels is independent of these traits. GWAS also concluded that the effect of the G6PC2 variation on FPG levels might be independent from adiposity-induced insulin resistance.

The rs560887 SNP is located close to the acceptor splice-site in the third intron of G6PC2 and predicted to affect pre-mRNA splicing by alternative usage of exon 4 $[13,14]$. Due to tissue-specific expression of various G6PC2 splice forms [21], the pancreatic RNA samples from individuals with different rs560887 genotypes (which are not readily available) would be necessary to directly test the latter hypothesis.

In conclusion, our results independently confirm the robust association of G6PC2/rs560887 (or another tightly linked, yet undiscovered variant) with FPG levels in nondiabetic NHWs and further support the relevance of the glucose phosphorylation pathway to blood glucose homeostasis in the general population. Although additional GWAS and meta-analysis increasingly support the association of G6PC2/rs560887 with FPG levels in individuals of European descent [22], our results further extend this observation to the Hispanic population by providing evidence for association that warrants replication in larger samples due to lower MAF of rs560887 in this population. The small effect sizes of G6PC2/rs560887 and other relevant genetic variants in GCKR, GCK and MTNR1B [22] suggest that the majority of genetic factors that regulate FPG levels remain to be identified. The absence (or very low MAF) of this SNP in our black African sample supports the presence of additional common variants (in G6PC2 and/or other genes) that contribute to blood glucose regulation in individuals from various ethnic origins. In fact, the rs560887 variant is also rare among Asians and a recent study demonstrated that another G6PC2 variant common in Chinese was associated with FPGlevels in this population [23]. Resequencing of G6PC2 in selected individuals with upper and lower range of FPG levels may help to determine the extent to which common and rare variation of G6PC2 explains the variation in FPG levels and whether rs560887 is the most important G6PC2-linked genetic determinant of glucoregulation.

\section{Acknowledgments}

We are grateful to the subjects who participated in this study. This study was supported in part by grants HL 84613 and HL 444313 from the National Heart, Lung, and Blood Institute.

\section{References}

1 Weedon MN, Clark VJ, Qian Y, et al: A common haplotype of the glucokinase gene alters fasting glucose and birth weight: association in six studies and population-genetics analyses. Am J Hum Genet 2006;79:991-1001.

-2 Boehnke M, Moll PP, Kottke BA, Weidman WH: Partitioning the variability of fasting plasma glucose levels in pedigrees: genetic and environmental factors. Am J Epidemiol 1987; 125:679-689.

-3 Henkin L, Bergman RN, Bowden DW, et al: Genetic epidemiology of insulin resistance and visceral adiposity: the IRAS Family Study design and methods. Ann Epidemiol 2003;13:211-217.

4 Pilia G, Chen WM, Scuteri A, et al: Heritability of cardiovascular and personality traits in 6,148 Sardinians. PLoS Genet 2006;2:e132.

-5 Bjørnholt JV, Erikssen G, Aaser E, et al: Fasting blood glucose: an underestimated risk factor for cardiovascular death. Results from a 22-year follow-up of healthy nondiabetic men. Diabetes Care 1999;22:45-49.
-6 Coutinho M, Gerstein HC, Wang Y, Yusuf S: The relationship between glucose and incident cardiovascular events: a metaregression analysis of published data from 20 studies of 95,783 individuals followed for 12.4 years. Diabetes Care 1999;22:233-240.

7 Wei M, Gibbons LW, Mitchell TL, et al: Low fasting plasma glucose level as a predictor of cardiovascular disease and all-cause mortality. Circulation 2000;101:2047-2052.

8 DECODE Study Group, European Diabetes Epidemiology Group: Is the current definition for diabetes relevant to mortality risk from all causes and cardiovascular and noncardiovascular diseases? Diabetes Care 2003; 26:688-696.

-9 Mukherjee R, Wagar D, Stephens TA, LeeChan E, Singh B: Identification of CD4+ T cell-specific epitopes of islet-specific glucose-6-phosphatase catalytic subunit-related protein: a novel beta cell autoantigen in type 1 diabetes. J Immunol 2005;174:53065315.
10 Unger WW, Pinkse GG, Mulder-van der Kracht S, et al: Human clonal CD8 autoreactivity to an IGRP islet epitope shared between mice and men. Ann NY Acad Sci 2007; 1103:192-195.

-11 Jarchum I, Nichol L, Trucco M, Santamaria P, DiLorenzo TP: Identification of novel IGRP epitopes targeted in type 1 diabetes patients. Clin Immunol 2008;127:359-365.

12 Wang Y, Martin CC, Oeser JK, et al: Deletion of the gene encoding the islet-specific glucose-6-phosphatase catalytic subunit-related protein autoantigen results in a mild metabolic phenotype. Diabetologia 2007;50: 774-778.

13 Bouatia-Naji N, Rocheleau G, Van Lommel $\mathrm{L}$, et al: A polymorphism within the G6PC2 gene is associated with fasting plasma glucose levels. Science 2008;320:1085-1088.

14 Chen WM, Erdos MR, Jackson AU, et al: Variations in the G6PC2/ABCB11 genomic region are associated with fasting glucose levels. J Clin Invest 2008;118:2620-2628. 
-15 Hamman RF, Marshall JA, Baxter J, et al: Methods and prevalence of non-insulin-dependent diabetes mellitus in a biethnic Colorado population. The San Luis Valley Diabetes Study. Am J Epidemiol 1989;129: 295-311.

16 Rewers M, Shetterly SM, Hoag S, et al: Is the risk of coronary heart disease lower in Hispanics than in non-Hispanic whites? The San Luis Valley Diabetes Study. Ethn Dis 1993;3:44-54.

-17 Kamboh MI, Rewers M, Aston CE, Hamman RF: Plasma apolipoprotein A-I, apolipoprotein $\mathrm{B}$, and lipoprotein(a) concentrations in normoglycemic Hispanics and non-Hispanic whites from the San Luis Valley, Colorado. Am J Epidemiol 1997;146:1011-1018.
8 Harris MR, Bunker CH, Hamman RF, et al: Racial differences in the distribution of a low density lipoprotein receptor-related protein (LRP) polymorphism and its association with serum lipoprotein, lipid and apolipoprotein levels. Atherosclerosis 1998;137:187195.

19 Genuth S, Alberti KG, Bennett P, et al: Fol low-up report on the diagnosis of diabetes mellitus. Diabetes Care 2003;26:3160-3167.

-20 Bunker CH, Ukoli FA, Okoro FI, et al: Correlates of serum lipids in a lean black population. Atherosclerosis 1996;123:215-225.
21 Dogra RS, Vaidyanathan P, Prabakar KR, et al: Alternative splicing of G6PC2, the gene coding for the islet-specific glucose-6-phosphatase catalytic subunit-related protein (IGRP), results in differential expression in human thymus and spleen compared with pancreas. Diabetologia 2006;49:953-957.

22 Prokopenko I, Langenberg C, Florez JC, et al: Variants in MTNR1B influence fasting glucose levels. Nat Genet 2009;41:77-81.

$23 \mathrm{Hu}$ C, Zhang R, Wang C, et al: A genetic variant of G6PC2 is associated with type 2 diabetes and fasting plasma glucose level in the Chinese population. Diabetologia 2009;52: 451-456. 